\title{
A PROSPECTIVE OBSERVATIONAL STUDY OF MINI-MENTAL STATE EXAMINATION IN ELDERLY PARTICIPANTS ATTENDING OUTPATIENT CLINIC IN GERIATRIC DEPARTMENT, MADRAS MEDICAL COLLEGE, CHENNAI
}

\author{
Kesavan Umakalyani', Manickam Senthilkumar ${ }^{2}$
}

${ }^{1}$ Assistant Professor, Department of Geriatric Medicine, Rajiv Gandhi Government General Hospital, Madras Medical College, Chennai. ${ }^{2}$ Assistant Professor, Department of Geriatric Medicine, Rajiv Gandhi Government General Hospital, Madras Medical College, Chennai.

ABSTRACT

\section{BACKGROUND}

The Mini-Mental State Examination (MMSE) or Folstein test is a 30-point questionnaire that is used extensively in clinical and research settings to measure cognitive impairment. It is commonly used in medicine and allied health to screen for dementia. It is also used to estimate the severity and progression of cognitive impairment and to follow the course of cognitive changes in an individual over time; thus making it an effective way to document an individual's response to treatment. The MMSE's purpose has been not, on its own, to provide a diagnosis for any particular disease.

The aim of the study is to observe the cognitive status of elderly patients attending Geriatric Outpatient clinic, Rajiv Gandhi Government General Hospital, Madras Medical College, Chennai using Folstein's Mini-Mental State Examination and study the same with respect to Gender and Education.

\section{MATERIALS AND METHODS}

Design- A hospital-based, prospective, observational study.

109 elderly patients were selected and Mini-mental State Examination (MMSE) was done. The Sample size was selected for convenience. Co-existing morbid conditions were also recorded.

\section{RESULTS}

In MMSE performance among elderly-

1. Out of 109 participants 68 participants had scored 25 and above, 29 participants had scores between 24 and 19 suggesting mild cognitive impairment and 12 had scores $\leq 18$ suggesting severe cognitive impairment.

2. There was no statistical difference between the two genders. ( $p>0.05)$.

3. Literates fared better than illiterates $(\mathrm{p}<0.05)$.

\section{CONCLUSION}

The validity of the MMSE should be considered with caution and confirmed with other relevant tests to assess cognitive function in the elderly population.

\section{KEYWORDS}

Mini-Mental Status Examination, Geriatric, Dementia, Elderly.

HOW TO CITE THIS ARTICLE: Umakalyani K, Senthilkumar M. A prospective observational study of mini-mental state examination in elderly participants attending outpatient clinic in geriatric department, Madras Medical College, Chennai. J. Evolution Med. Dent. Sci. 2018;7(03):315-320, DOI: 10.14260/jemds/2018/70

\section{BACKGROUND}

Cognition is the ability to interact meaningfully to one's environment.(1) There are many domains in cognition like attention, memory, language, visuospatial skills and executive functioning, each served by different parts of brain. Cognitive impairment increases with age.(2) In normal ageing the cognitive domains are affected in the following ways:

Attention- Sustained and primary attention span is preserved while there is impairment of divided attention.

Executive Function- There is difficulty in learning novel executive tasks.

'Financial or Other Competing Interest': None.

Submission 17-10-2017, Peer Review 25-12-2017,

Acceptance 01-01-2018, Published 13-01-2018.

Corresponding Author:

Kesavan Umakalyani,

Assistant Professor,

Department of Geriatric Medicine,

Rajiv Gandhi Government General Hospital,

Madras Medical College, Chennai.

E-mail: uma_bullu@yahoo.com

DOI: $10.14260 /$ jemds $/ 2018 / 70$

\section{Memory}

Remote memory, procedural memory and semantic call are all preserved while there is impairment in learning and recall of new memory.

\section{Language}

Comprehension, vocabulary and syntactic abilities are preserved while spontaneous word finding and verbal fluency is impaired.

There is slowness in information processing and reaction time.

Cognitive impairment is a spectrum which proceeds from that of Normal ageing, to Minimal Cognitive Impairment (MCI) which can be subjective and then becomes objective but without affecting the functional status and then progressing to Dementia. Precipitous decline can occur with some illness and drugs. (3)

But even though there is age-related impairment in cognition across multiple domains in healthy ageing, it is adequate to be functionally independent. (4)

Cognitive impairment is universally screened using Minimental State Examination (MMSE).(5) There are many other tools to screen cognition but MMSE is widely used and simple 
and easy to apply. MMSE briefly assesses all the following cognitive domains:

\begin{tabular}{|c|c|}
\hline Domain & Maximum Points \\
\hline Attention & 5 \\
\hline Orientation to time & 5 \\
\hline Orientation to place & 5 \\
\hline Registration & 3 \\
\hline Recall & 3 \\
\hline Naming & 2 \\
\hline Reading & 1 \\
\hline Writing & 1 \\
\hline Repetition & 1 \\
\hline 3 Stage command & 3 \\
\hline Copying & 1 \\
\hline Maximum Total points & 30 \\
\hline
\end{tabular}

It has a maximum score of 30 . A cut-off below 24 is mild cognitive impairment and below 18 is severe cognitive impairment.(1) But MMSE has its limitations. It is found to vary with culture, education and language. It has a "Ceiling effect"-even though cognitively impaired, highly educated individuals score high and "Floor effect" - cognitively intact individuals who are illiterate score low.(6) Normal tests reduce the probability of Dementia and abnormal tests increase the likelihood of Dementia but in itself MMSE score is not diagnostic in confirming or ruling out Dementia.(7)

There are many impediments in assessing cognitive status in elderly.(8) Sensory impairments such as vision and hearing impairment will make it difficult for elderly to perform the tasks. There is age-related psychomotor slowing and so it takes a longer time for an old individual to complete a task than his younger counterpart. Many comorbid conditions which are found increasingly in elderly like Diabetes, Hypertension, Hypothyroidism, Depression, Nutritional deficiency affects cognition. Stroke and Parkinson's disease which are diseases of elderly causes cognitive impairment. Essential tremor of ageing may impair the ability to draw and write. Polypharmacy is common among elderly. Drugs both prescribed and over-the-counter affect cognition.

"Delirium" or "Acute Confusional State" is one among the "Geriatric Giants" of clinical presentations.(6) Elderly people with any underlying disease may present with the clouded consciousness. Unless the baseline functional status is already known it is difficult to assess the cognitive status of the elderly who is seen in emergency department in delirious state. Also cognitive impairment may go unrecognised in elderly and may be unmasked by illness such as infection or metabolic disturbances.(7) So baseline screening of cognition is all the more important in elderly. Comprehensive Geriatric Assessment includes cognitive assessment a necessary part.(9)

The primary objective of this study is to assess the minimental status profile of patients in geriatric clinic in our hospital.

\section{Aim of the Study}

To observe the cognitive status of functionally independent elderly participants attending Geriatric Outpatient clinic and compare it among Gender and literacy.

\section{MATERIALS AND METHODS}

109 elderly patients (51 male and 58 female) attending the geriatric outpatient clinic of Department of Geriatric Medicine, Rajiv Gandhi Government General Hospital, Madras Medical College, Chennai were selected. The Sample size was selected for convenience. After obtaining approval from institution ethics committee informed consent was obtained from each individual and subjected to Folstein's Mini-mental State examination.

\section{Inclusion Criteria}

Willing participants above 60 years attending Geriatric outpatient clinic, Rajiv Gandhi Government General Hospital, Madras Medical College, Chennai

\section{Exclusion Criteria}

History of stroke, dementia, alcoholism, chronic intake of drugs affecting cognition, severe visual and hearing impairment.

The selected participants were subjected to the test by a trained social health worker and the data were documented and entered in MS Excel sheet. Date of birth of the participants were confirmed with their available government identification records. If not available it was approximately calculated with their age at marriage and age of their first child. MMSE was appropriately translated and explained in their local language and applied. Comorbidities were recorded. Functional status was noted. Only functionally independent elders were taken for study to rule out frank Dementia.

\section{Statistical Analysis}

For continuous data, the descriptive statistics n, Mean, SD, Median, IQR, Minimum and Maximum was presented. For categorical data, the number of patients and percentage was presented. Based on the normality of data, the parametric $t$ test was applied to the data. All tests were two-sided at $\alpha=0.05$ level of significance. All analyses were done using Statistical Package for Social Services (SPSS) software Version 21.0 (Armonk, NY: IBM Corp).

\section{RESULTS}

\begin{tabular}{|c|c|c|c|}
\hline & & Age (Years) & MMSE Score \\
\hline Total $n=$ & & & \\
\hline Mea & & 68.25 & 25.30 \\
\hline Medi & & 67.00 & 27.00 \\
\hline Std. Dev & ion & 6.475 & 4.371 \\
\hline Minim & & 60 & 13 \\
\hline Maxim & & 92 & 30 \\
\hline & 25 & 64.00 & 23.00 \\
\hline Percentiles & 50 & 67.00 & 27.00 \\
\hline & 75 & 71.50 & 29.00 \\
\hline
\end{tabular}

Minimum age in our study group was 60 and maximum age studied was 92. Minimum MMSE score was 13 and Maximum score was 30. 


\begin{tabular}{|c|c|c|}
\hline MMSE Score & Frequency $(\mathrm{n}=)$ & Percent (\%) \\
\hline 18 and below & 12 & 11.0 \\
\hline 19 to 24 & 29 & 26.6 \\
\hline 25 and above & 68 & 62.4 \\
\hline Total & 109 & 100.0 \\
\hline
\end{tabular}

In our study group, 12 participants (11\%) scored below or equal to 18 in MMSE, 29 participants (26.6\%) scored between 19 - 24 and 68 participants (62.4\%) scored 25 and above-

\begin{tabular}{|c|c|c|c|}
\hline MMSE Score & $\begin{array}{c}\text { Degree of } \\
\text { Cognitive } \\
\text { impairment }\end{array}$ & $\begin{array}{c}\text { No. of } \\
\text { participants } \\
\text { (n=) }\end{array}$ & $\begin{array}{c}\text { Percentage } \\
\text { (\%) }\end{array}$ \\
\hline $30-25$ & Nil & 68 & 62.4 \\
\hline $24-19$ & Mild & 29 & 26.6 \\
\hline $18 \&$ less & Severe & 12 & 11 \\
\hline \multicolumn{4}{|r|}{ Table 3. MMSE Score and Cognitive Impairment } \\
\hline
\end{tabular}

\begin{tabular}{|c|c|c|c|}
\hline \multicolumn{2}{|c|}{ Gender } & Frequency (n=) & Percent (\%) \\
\hline \multirow{4}{*}{} & Male & 51 & 46.8 \\
\cline { 2 - 4 } & Female & 58 & 53.2 \\
\cline { 2 - 4 } & Total & $\mathbf{1 0 9}$ & $\mathbf{1 0 0 . 0}$ \\
\hline \multicolumn{3}{|c|}{ Table 4. Gender Distribution } \\
\hline
\end{tabular}

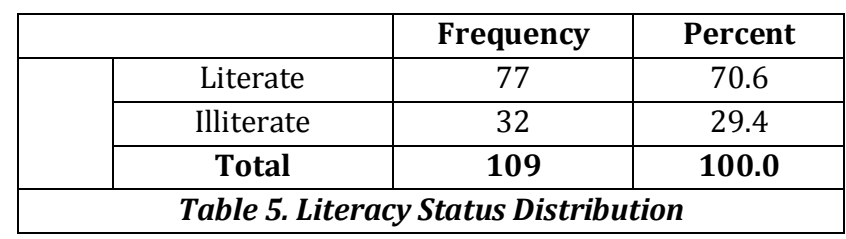

\begin{tabular}{|c|c|c|}
\hline Educational Level & $\begin{array}{c}\text { Frequency } \\
(n=)\end{array}$ & Percent (\%) \\
\hline 10th & 19 & 17.4 \\
\hline $1^{\text {st }}$ & 3 & 2.8 \\
\hline $2^{\text {nd }}$ & 9 & 8.3 \\
\hline $3^{\text {rd }}$ & 1 & 0.9 \\
\hline $4^{\text {th }}$ & 5 & 4.6 \\
\hline $5^{\text {th }}$ & 13 & 11.9 \\
\hline $6^{\text {th }}$ & 5 & 4.6 \\
\hline $7^{\text {th }}$ & 3 & 2.8 \\
\hline $8^{\text {th }}$ & 12 & 11.0 \\
\hline $9^{\text {th }}$ & 3 & 2.8 \\
\hline Diploma & 1 & 0.9 \\
\hline Graduate & 3 & 2.8 \\
\hline Illiterate & 32 & 29.4 \\
\hline Total & 109 & 100.0 \\
\hline \multicolumn{3}{|c|}{ Table 6. Education Level Distribution } \\
\hline
\end{tabular}

\begin{tabular}{|c|c|c|c|}
\hline \multicolumn{2}{|c|}{ Functional Status } & Frequency (n=) & Percent (\%) \\
\hline \multirow{4}{*}{} & & & \\
\cline { 2 - 4 } & Independent & 109 & 100.0 \\
\cline { 2 - 4 } & Total & 109 & 100.0 \\
\hline \multicolumn{3}{|c|}{ Table 7. Functional Status Distribution } \\
\hline
\end{tabular}

\begin{tabular}{|c|c|c|c|c|c|}
\hline Gender & $\mathbf{N}$ & $\begin{array}{l}\text { Mean } \\
\text { MMSE }\end{array}$ & $\begin{array}{c}\text { Std. } \\
\text { Deviation }\end{array}$ & $\begin{array}{c}\text { Std. } \\
\text { Error } \\
\text { Mean }\end{array}$ & P value \\
\hline Male & 51 & 26.16 & 3.844 & .538 & 0.0546 \\
\hline Female & 58 & 24.55 & 4.691 & .616 & \\
\hline
\end{tabular}

There is no statistical difference in the MMSE performance between either sexes ( $p>0.05)$.

\begin{tabular}{|c|c|c|c|c|c|}
\hline Education & N & $\begin{array}{c}\text { Mean } \\
\text { MMSE }\end{array}$ & $\begin{array}{c}\text { Std. } \\
\text { Deviation }\end{array}$ & $\begin{array}{c}\text { Std. } \\
\text { Error } \\
\text { Mean }\end{array}$ & P value \\
\hline Literate & 77 & 27.47 & 2.269 & .259 & $<0.0001$ \\
\hline Illiterate & 32 & 20.09 & 3.779 & .668 & \\
\hline \multicolumn{7}{|c|}{ Table 9. Literacy and MMSE Performance } \\
\hline
\end{tabular}

MMSE performance is better among literates than among Illiterates. $(\mathrm{p}<0.05)$.
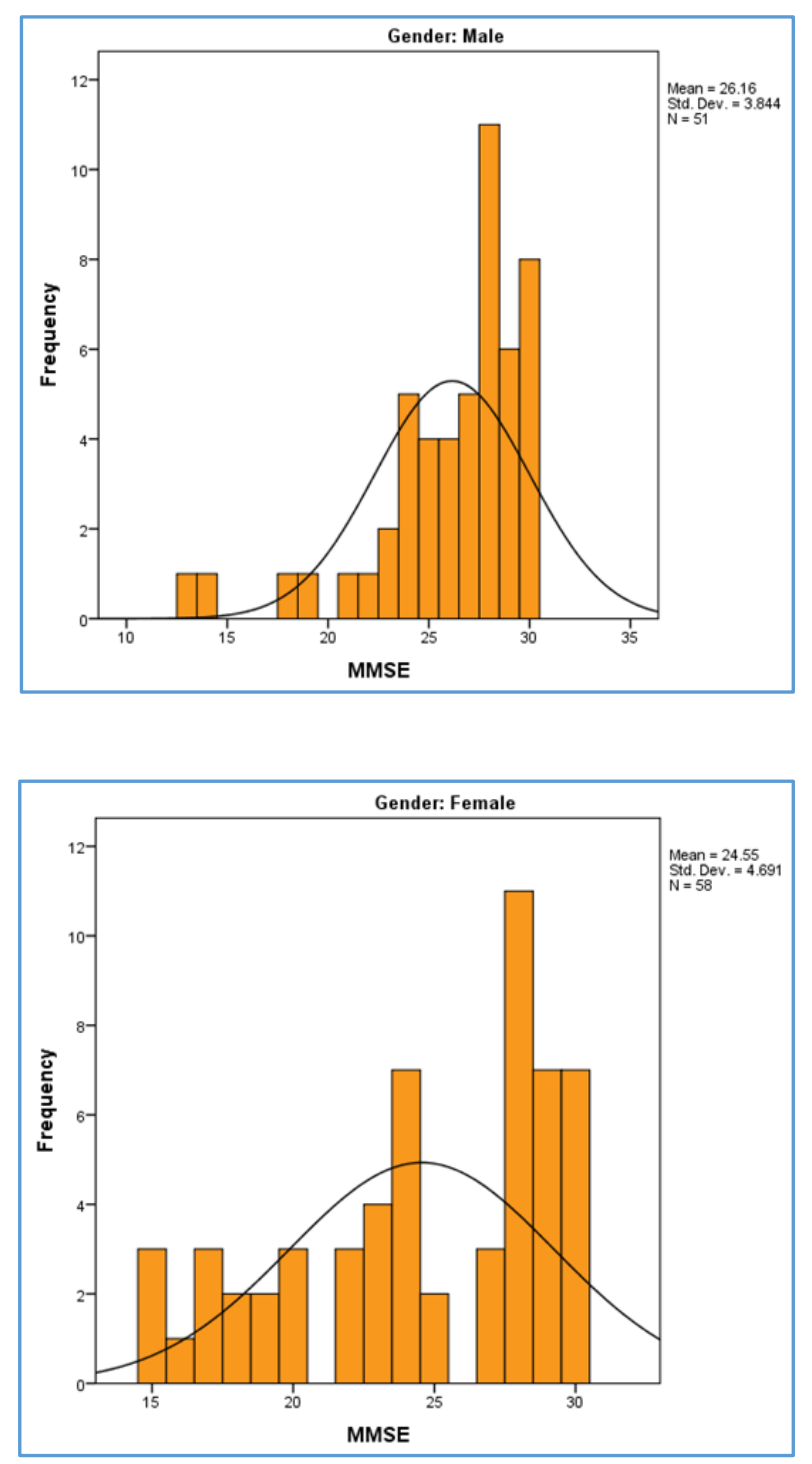

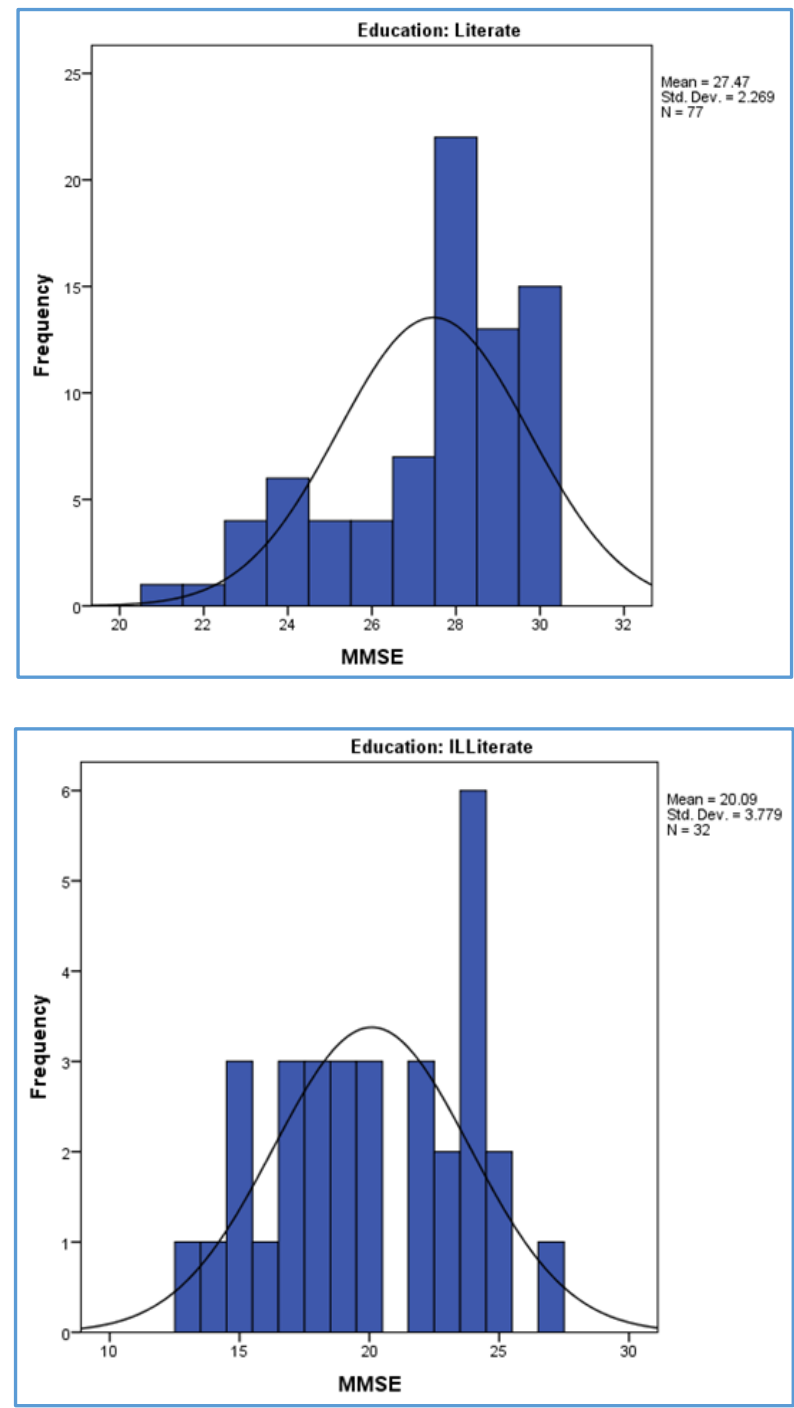

T-Test

\begin{tabular}{|c|c|c|c|c|c|}
\hline \multicolumn{2}{|c|}{ Gender } & N & Mean & $\begin{array}{c}\text { Std. } \\
\text { Deviation }\end{array}$ & $\begin{array}{c}\text { Std. Error } \\
\text { Mean }\end{array}$ \\
\hline \multirow{2}{*}{ MMSE } & Male & 51 & 26.16 & 3.844 & 0.538 \\
\cline { 2 - 6 } & Female & 58 & 24.55 & 4.691 & 0.616 \\
\hline & \multicolumn{5}{|c}{ Group Statistics } \\
\hline
\end{tabular}

\begin{tabular}{|c|c|c|c|c|c|}
\hline & \multicolumn{2}{|c|}{$\begin{array}{c}\text { Levene's Test } \\
\text { for Equality of } \\
\text { Variances }\end{array}$} & \multicolumn{2}{|c|}{$\begin{array}{c}\text { t-test for } \\
\text { Equality of } \\
\text { Means } \\
\end{array}$} \\
\hline & & $\mathbf{F}$ & Sig. & $\mathbf{t}$ & df \\
\hline \multirow{2}{*}{ MMSE } & $\begin{array}{c}\text { Equal } \\
\text { variances } \\
\text { assumed }\end{array}$ & 5.743 & 0.018 & 1.937 & 107 \\
\hline & $\begin{array}{c}\text { Equal } \\
\text { variances not } \\
\text { assumed }\end{array}$ & & & 1.962 & 106.496 \\
\hline
\end{tabular}

\begin{tabular}{|c|c|c|c|c|c|}
\hline & \multicolumn{4}{|c|}{ t-test for Equality of Means } \\
\hline & & \multirow[t]{2}{*}{$\begin{array}{l}\text { Sig. } \\
(2- \\
\text { tailed) }\end{array}$} & \multirow[t]{2}{*}{$\begin{array}{c}\text { Mean } \\
\text { Difference }\end{array}$} & \multirow[t]{2}{*}{$\begin{array}{l}\text { Std. Error } \\
\text { Difference }\end{array}$} & $\begin{array}{c}95 \% \\
\text { Confidence } \\
\text { Interval of } \\
\text { the } \\
\text { Difference }\end{array}$ \\
\hline & & & & & Lower \\
\hline \multirow[b]{2}{*}{ MMSE } & $\begin{array}{c}\text { Equal } \\
\text { variances } \\
\text { assumed }\end{array}$ & 0.055 & 1.605 & 0.828 & -0.037 \\
\hline & $\begin{array}{c}\text { Equal } \\
\text { variances } \\
\text { not } \\
\text { assumed }\end{array}$ & 0.052 & 1.605 & 0.818 & -0.017 \\
\hline & & Indep & lent Samp & Test & \\
\hline
\end{tabular}

\begin{tabular}{|c|c|c|}
\hline \multicolumn{2}{|c|}{} & $\begin{array}{c}\text { t-test for } \\
\text { Equality of } \\
\text { Means }\end{array}$ \\
\cline { 3 - 3 } & $\begin{array}{c}\text { 95\% Confidence } \\
\text { Interval of the } \\
\text { Difference }\end{array}$ \\
\cline { 3 - 3 } & Upper \\
\hline MMSE & Equal variances assumed & 3.248 \\
\cline { 3 - 3 } & Equal variances not assumed & 3.227 \\
\hline \multicolumn{2}{|c|}{ Independent Samples Test } \\
\hline
\end{tabular}

\section{T-Test}

\begin{tabular}{|c|c|c|c|c|c|}
\hline & Education & N & Mean & $\begin{array}{c}\text { Std. } \\
\text { Deviation }\end{array}$ & $\begin{array}{c}\text { Std. Error } \\
\text { Mean }\end{array}$ \\
\hline \multirow{2}{*}{ MMSE } & Literate & 77 & 27.47 & 2.269 & 0.259 \\
\cline { 2 - 6 } & Illiterate & 32 & 20.09 & 3.779 & 0.668 \\
\hline \multicolumn{7}{|c|}{ Group Statistics } \\
\hline
\end{tabular}

\begin{tabular}{|c|c|c|c|c|c|}
\hline & \multicolumn{2}{|c|}{$\begin{array}{c}\text { Levene's Test for } \\
\text { Equality of } \\
\text { Variances }\end{array}$} & \multicolumn{2}{|c|}{$\begin{array}{l}\text { t-test for } \\
\text { Equality of } \\
\text { Means }\end{array}$} \\
\hline & & $\mathbf{F}$ & Sig. & $\mathbf{t}$ & Df \\
\hline \multirow{2}{*}{$\sum_{\Sigma}^{5}$} & $\begin{array}{c}\text { Equal variances } \\
\text { assumed }\end{array}$ & 19.714 & 0.000 & 12.558 & 107 \\
\hline & $\begin{array}{c}\text { Equal variances } \\
\text { not assumed }\end{array}$ & & & 10.294 & 40.610 \\
\hline
\end{tabular}

\begin{tabular}{|c|c|c|c|c|c|}
\hline & \multicolumn{4}{|c|}{ t-test for Equality of Means } \\
\hline & & \multirow[t]{2}{*}{\begin{tabular}{c|} 
Sig. \\
$(2-$ \\
tailed)
\end{tabular}} & \multirow[t]{2}{*}{$\begin{array}{c}\text { Mean } \\
\text { Differenc } \\
\text { e }\end{array}$} & \multirow[t]{2}{*}{$\begin{array}{c}\text { Std. Error } \\
\text { Differenc } \\
\text { e }\end{array}$} & $\begin{array}{c}95 \% \\
\text { Confidenc } \\
\text { e Interval } \\
\text { of the } \\
\text { Difference } \\
\end{array}$ \\
\hline & & & & & Lower \\
\hline \multirow{3}{*}{$\begin{array}{c}\text { MMS } \\
\text { E }\end{array}$} & Equal & 0.00 & \multirow[t]{2}{*}{7.374} & \multirow[t]{2}{*}{0.587} & \multirow[t]{2}{*}{6.210} \\
\hline & $\begin{array}{l}\text { variance } \\
\text { s } \\
\text { assumed }\end{array}$ & 0 & & & \\
\hline & $\begin{array}{c}\text { Equal } \\
\text { variance } \\
\text { s not } \\
\text { assumed }\end{array}$ & $\begin{array}{c}0.00 \\
0\end{array}$ & 7.374 & 0.716 & 5.927 \\
\hline
\end{tabular}




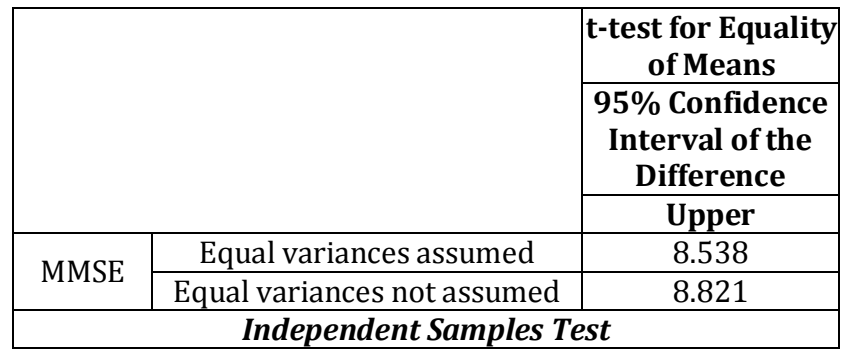

Summary

\begin{tabular}{|c|c|c|c|}
\hline \multicolumn{2}{|c|}{} & Age (years) & MMSE \\
\hline \multirow{2}{*}{$\mathbf{N}$} & Valid & $\mathbf{1 0 9}$ & $\mathbf{1 0 9}$ \\
\cline { 2 - 4 } & Missing & $\mathbf{0}$ & $\mathbf{0}$ \\
\hline \multicolumn{2}{|c|}{ Mean } & 68.25 & 25.30 \\
\hline \multicolumn{2}{|c|}{ Median } & 67.00 & 27.00 \\
\hline \multicolumn{2}{|c|}{ Std. Deviation } & 6.475 & 4.371 \\
\hline \multicolumn{2}{|c|}{ Minimum } & 60 & 13 \\
\hline \multicolumn{2}{|c|}{ Maximum } & 92 & 30 \\
\hline \multirow{3}{*}{ Percentiles } & 25 & 64.00 & 23.00 \\
\cline { 2 - 4 } & 50 & 67.00 & 27.00 \\
\cline { 2 - 4 } & 75 & 71.50 & 29.00 \\
\hline \multicolumn{2}{|c|}{ Statistics } \\
\hline
\end{tabular}

\section{Frequency Table}

\begin{tabular}{|c|c|c|c|c|c|}
\hline \multicolumn{2}{|c|}{} & Frequency & Percent & $\begin{array}{c}\text { Valid } \\
\text { Percent }\end{array}$ & $\begin{array}{c}\text { Cumulative } \\
\text { Percent }\end{array}$ \\
\hline \multirow{3}{*}{ Valid } & Male & 51 & 46.8 & 46.8 & 46.8 \\
\cline { 2 - 6 } & Female & 58 & 53.2 & 53.2 & 100.0 \\
\cline { 2 - 6 } & Total & $\mathbf{1 0 9}$ & $\mathbf{1 0 0 . 0}$ & $\mathbf{1 0 0 . 0}$ & \\
\hline \multicolumn{6}{|c}{ Gender } \\
\hline
\end{tabular}

\begin{tabular}{|c|c|c|c|c|c|}
\hline \multicolumn{2}{|c|}{} & Frequency & Percent & $\begin{array}{c}\text { Valid } \\
\text { Percent }\end{array}$ & $\begin{array}{c}\text { Cumulative } \\
\text { Percent }\end{array}$ \\
\hline \multirow{3}{*}{ Valid } & Literate & 77 & 70.6 & 70.6 & 70.6 \\
\cline { 2 - 6 } & Illiterate & 32 & 29.4 & 29.4 & 100.0 \\
\cline { 2 - 6 } & Total & $\mathbf{1 0 9}$ & $\mathbf{1 0 0 . 0}$ & $\mathbf{1 0 0 . 0}$ & Education \\
\hline & \multicolumn{5}{|c|}{} \\
\hline
\end{tabular}

\begin{tabular}{|c|c|c|c|c|c|}
\hline & Frequency & Percent & $\begin{array}{c}\text { Valid } \\
\text { Percent }\end{array}$ & $\begin{array}{c}\text { Cumulative } \\
\text { Percent }\end{array}$ \\
\hline \multirow{14}{*}{ : } & 10th & 19 & 17.4 & 17.4 & 17.4 \\
\hline & $1^{\text {st }}$ & 3 & 2.8 & 2.8 & 20.2 \\
\hline & $2^{\text {nd }}$ & 9 & 8.3 & 8.3 & 28.4 \\
\hline & $3^{\text {rd }}$ & 1 & 0.9 & 0.9 & 29.4 \\
\hline & $4^{\text {th }}$ & 5 & 4.6 & 4.6 & 33.9 \\
\hline & $5^{\text {th }}$ & 13 & 11.9 & 11.9 & 45.9 \\
\hline & $6^{\text {th }}$ & 5 & 4.6 & 4.6 & 50.5 \\
\hline & $7^{\text {th }}$ & 3 & 2.8 & 2.8 & 53.2 \\
\hline & $8^{\text {th }}$ & 12 & 11.0 & 11.0 & 64.2 \\
\hline & $9^{\text {th }}$ & 3 & 2.8 & 2.8 & 67.0 \\
\hline & Diploma & 1 & 0.9 & 0.9 & 67.9 \\
\hline & Graduate & 3 & 2.8 & 2.8 & 70.6 \\
\hline & Illiterate & 32 & 29.4 & 29.4 & 100.0 \\
\hline & Total & 109 & 100.0 & 100.0 & \\
\hline
\end{tabular}

\begin{tabular}{|c|c|c|c|c|c|}
\hline & \multirow{2}{*}{\begin{tabular}{|c|} 
Frequency \\
3
\end{tabular}} & \multirow{2}{*}{$\begin{array}{l}\% \\
2.8\end{array}$} & \multirow{2}{*}{\begin{tabular}{|l|}
$\begin{array}{c}\text { Valid } \\
\%\end{array}$ \\
2.8 \\
\end{tabular}} & \multirow{2}{*}{\begin{tabular}{|c|}
$\begin{array}{c}\text { Cumulative } \\
\%\end{array}$ \\
2.8 \\
\end{tabular}} \\
\hline \multirow{5}{*}{ 국 } & & & & & \\
\hline & Anaemia & 1 & 0.9 & 0.9 & \begin{tabular}{|l|}
3.7 \\
\end{tabular} \\
\hline & CAD & 9 & 8.3 & 8.3 & 11.9 \\
\hline & CAD, COPD & 2 & 1.8 & 1.8 & 13.8 \\
\hline & $\begin{array}{c}\text { CAD, COPD, DM, } \\
\text { SHT }\end{array}$ & 1 & 0.9 & 0.9 & 14.7 \\
\hline
\end{tabular}

\begin{tabular}{|c|c|c|c|c|}
\hline $\begin{array}{c}\text { CAD, } \\
\text { Hypothyroidism }\end{array}$ & 1 & 0.9 & 0.9 & 15.6 \\
\hline CKD & 1 & 0.9 & 0.9 & 16.5 \\
\hline COPD & 4 & 3.7 & 3.7 & 20.2 \\
\hline $\mathrm{DM}$ & 13 & 11.9 & 11.9 & 32.1 \\
\hline DM, CAD & 7 & 6.4 & 6.4 & 38.5 \\
\hline DM, HT & 2 & 1.8 & 1.8 & 40.4 \\
\hline $\begin{array}{c}\text { DM, HT, CAD, } \\
\text { Hypothyroidism }\end{array}$ & 1 & 0.9 & 0.9 & 41.3 \\
\hline DM, OA knees & 1 & 0.9 & 0.9 & 42.2 \\
\hline DM, SHT & 6 & 5.5 & 5.5 & 47.7 \\
\hline $\begin{array}{c}\text { DM, SHT, BA, } \\
\text { Hypothyroidism }\end{array}$ & 1 & 0.9 & 0.9 & 48.6 \\
\hline DM, SHT, CAD & 3 & 2.8 & 2.8 & 51.4 \\
\hline $\begin{array}{c}\text { DM, SHT, } \\
\text { Hypothyroidism }\end{array}$ & 1 & 0.9 & 0.9 & 52.3 \\
\hline $\begin{array}{c}\text { DM, HT, } \\
\text { Hypothyroidism }\end{array}$ & 1 & 0.9 & 0.9 & 53.2 \\
\hline Dyslipidaemia & 1 & 0.9 & 0.9 & 54.1 \\
\hline Nil & 8 & 7.3 & 7.3 & 61.5 \\
\hline Osteoarthritis & 1 & 0.9 & 0.9 & 62.4 \\
\hline Parkinson's, CAD & 1 & 0.9 & 0.9 & 63.3 \\
\hline RA, CAD, SHT & 1 & 0.9 & 0.9 & 64.2 \\
\hline SHT & 11 & 10.1 & 10.1 & 74.3 \\
\hline SHT, BA, CAD & 1 & 0.9 & 0.9 & 75.2 \\
\hline SHT, CAD & 8 & 7.3 & 7.3 & 82.6 \\
\hline $\begin{array}{c}\text { SHT, CAD, BA, } \\
\text { Hypothyroidism }\end{array}$ & 1 & 0.9 & 0.9 & 83.5 \\
\hline SHT, CKD & 2 & 1.8 & 1.8 & 85.3 \\
\hline $\begin{array}{c}\text { SHT, DIABETES, } \\
\text { CAD }\end{array}$ & 1 & 0.9 & 0.9 & 86.2 \\
\hline SHT, DM & 5 & 4.6 & 4.6 & 90.8 \\
\hline SHT, DM, CAD & 6 & 5.5 & 5.5 & 96.3 \\
\hline $\begin{array}{c}\text { SHT, DM, CAD, } \\
\text { Hypothyroidism }\end{array}$ & 1 & 0.9 & 0.9 & 97.2 \\
\hline SHT, DM, CKD & 1 & 0.9 & 0.9 & 98.2 \\
\hline $\begin{array}{c}\text { SHT, DM, } \\
\text { Dyslipidaemia }\end{array}$ & 1 & 0.9 & 0.9 & 99.1 \\
\hline $\begin{array}{c}\text { SHT, } \\
\text { Dyslipidaemia }\end{array}$ & 1 & 0.9 & 0.9 & 100.0 \\
\hline Total & 109 & 100.0 & 100.0 & \\
\hline \multicolumn{5}{|c|}{ Comorbidities } \\
\hline
\end{tabular}

\begin{tabular}{|c|c|c|c|c|c|}
\hline \multicolumn{2}{|c|}{} & Frequency & Percent & $\begin{array}{c}\text { Valid } \\
\text { Percent }\end{array}$ & $\begin{array}{c}\text { Cumulative } \\
\text { Percent }\end{array}$ \\
\hline & Independent & 109 & 100 & 100 & 100.0 \\
\cline { 2 - 5 } & Total & $\mathbf{1 0 9}$ & $\mathbf{1 0 0 . 0}$ & $\mathbf{1 0 0 . 0}$ & \\
\hline & \multicolumn{6}{|c|}{ Functional Status } \\
\hline
\end{tabular}

\section{DISCUSSION}

1. All the participants in this study were functionally independent elderly people. However, MMSE performance in our participants suggested a percentage of them to have varying degrees of cognitive impairment.

2. There is no statistical difference between males and females in the performance of MMSE. This was in accordance with earlier studies.(3)

3. Literates fared better than the illiterates. 


\section{CONCLUSION}

The validity of the MMSE should be considered with caution and should be confirmed with other relevant tests to assess cognitive function in the elderly population.

\section{REFERENCES}

[1] Folstein MF, Folstein SE, McHugh PR. "Mini-mental state". A practical method for grading the cognitive state of patients for the clinician. J Psychiatr Res 1975;12(3):189-98.

[2] Multicultural issues in neuropsychological assessment, Alzheimer's Dementia. Symposia S2 - 01, July 16, 2012.

[3] Piccinin AM, Muniz-Terrera G, Clouston S, et al. Coordinate analysis of age, sex and education effects on change in MMSE scores. J Gerontol B Psychol Sci Soc Sci 2013;68(3):374-90.

[4] Shim YS, Yang DW, Kim HJ, et al. Characteristic differences in the Mini-mental state examination used in Asian countries. BMC Neurol 2017;17:141.
[5] Morley JE. A brief history of geriatrics. J Gerontol A Biol Sci Med Sci 2004;59(11):1132-52.

[6] Morley JE, Morris JC, Berg-Weger M, et al. Brain health: the importance of recognizing cognitive impairment: an IAGG consensus conference. J Am Med Dir Assoc 2015;16(9):731-9.

[7] D'cruz SM, Rajaratnam N. The effect of aging on cognitive function in a South Indian population. International Journal of Scientific and Research Publications 2013;3(5):1-5.

[8] Tombaugh TN, Mclntyre NJ. The mini-mental state examination: a comprehensive review. J Am Geriatr Soc 1992;40(9):922-35.

[9] Jacqmin-Gadda H, Fabrigoule C, Commenges D, et al. A 5-year longitudinal study of the mini-mental state examination in normal aging. Am J Epidemiol 1997;145(6):498-506. 